\title{
Die GEMA bleibt außen vor!
}

Rundfunkgebühren fallen pro Praxis - nicht pro Praxisinhaber - an. Die GEMA-Gebühren für Arztpraxen sind jedoch nicht rechtens. Zahlungsaufforderungen der GEMA sollten umgehend zurückgeschickt und als unberechtigt gekennzeichnet werden. Sollten Sie aus Unkenntnis bereits einen Vertrag mit der GEMA eingegangen sein, können Sie diesen fristlos kündigen.

Wer im Wartezimmer Musik abspielt, ist nicht zu einer Zahlung an die Gesellschaft für musikalische Aufführungsund mechanische Vervielfältigungsrechte (GEMA) verpflichtet. Dennoch kommt es vor, dass Zahlungsaufforderungen verschickt werden. Mehrere Urteile des Europäischen Gerichtshofs (EuGH) beziehungsweise des Landgerichts Köln und des Amtsgerichts Düsseldorf existieren.

\section{Öffentlichkeit fehlt}

Der EuGH hatte entschieden, dass im Falle einer italienischen Zahnarztpraxis die Hintergrundmusik nicht GEMApflichtig ist.

Das Landgericht Köln wies die Klage der GEMA gegen eine physiotherapeutische Praxis ab, bei der im Rezeptionsbereich Radio gehört wurde. Nach dem Urteil der Kölner Richter handelte es sich bei den Zuhörern um eine klar abgrenzbare private Gruppe von Personen, weil andere Personen grundsätzlich keinen Zutritt zur Behandlung in der Praxis hatten. Damit fehle es am Merkmal der Öffentlichkeit. Außerdem erreicht die Zahl der in der Praxis anwesenden Personen nicht eine Menge, bei der man von Öffentlichkeit sprechen kann. Drittens dient das Abspielen von Hintergrundmusik in einer Praxis nicht einem Erwerbszweck wie beispielsweise in Hotels oder Gaststätten. Die zu Gehör gebrachte Musik sei nicht geeignet, sich auf das Einkommen des Praxisinhabers positiv auswirken. Die Patienten seien dieser Musik zufällig ausgesetzt, weil sie als Hauptzweck ihres Besuches physiotherapeutische Leistungen erhalten möchten. Einer Musikdarbietung beizuwohnen entspreche nicht der Motivation zum Praxisbesuch.

Das Amtsgericht Düsseldorf entschied den Fall eines Zahnarztes, der aus
Unkenntnis 2003 einen Vertrag mit der GEMA eingegangen war. Dieser sei einem sogenannten Motivirrtum bei Abschluss des Vertrages unterlegen. Deshalb musste die GEMA eine Teilrückzahlung an den Zahnarzt leisten. Der Vertrag konnte sofort aufgelöst werden.

\section{Rundfunkgebühr ist Pflicht}

Die bereits entschiedenen Vorgänge zeigen, dass man als Praxisinhaber nicht genug aufpassen kann, um ungerechtfertigte Kosten zu vermeiden. GEMAZahlungsaufforderungen sollten also umgehend zurückgeschickt und als unberechtigt gekennzeichnet werden. Bereits bestehende GEMA-Verträge können fristlos gekündigt werden.

Vorsicht ist in diesem Zusammenhang auch angebracht bei unaufgefordert zugesandten Rechnungen zu Telefonbuchoder Branchenverzeichniseinträgen für Ärzte. Allenfalls kann bei genauester Prüfung eventuell eine Anzeige im örtlichen Telefonbuch geschaltet werden, wobei Ärzte nach Fachgruppen sortiert ohnehin kostenfrei im normalen Telefonbuchlayout abgedruckt werden.

Den Rundfunkgebühren der öffentlich-rechtlichen Fernseh- und Rundfunkanstalten kann man jedoch nicht entgehen. Auch eine Arztpraxis ist zahlungspflichtig. Aber auch hier ist genauestens zu prüfen, ob nicht zu viele Gebühren erhoben werden. In Gemeinschaftspraxen beispielsweise fällt die Rundfunkgebühr praxisbezogen nur einmal an, nicht für jeden einzelnen Teilhaber separat.

Differenzierte Bestimmungen gibt es auch für Dienst-PKW oder für eine Betriebsstätte in der privaten Wohnungsanschrift, wo gegebenenfalls zusammen mit dem einzigen PKW nur eine Gesamtgebühr anfällt.

Dr. med. Gunther Carl, Kitzingen 